\title{
Offene Fragen
}

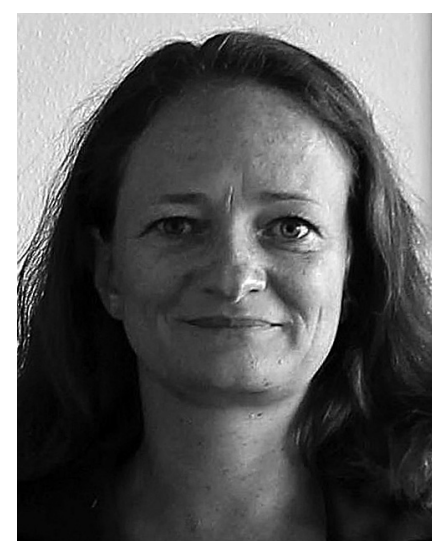

VON KATRIN SCHNEIDERS Prof. Dr. Katrin Schneiders ist Hochschullehrerin für Wissenschaft der Sozialen Arbeit mit Schwerpunkt Sozialwirtschaft an der Hochschule Koblenz. Nach beruflicher Tätigkeit in der anwendungsbezogenen Forschung und Beratung promovierte sie 2009 an der Ruhr-Universität Bochum mit einer Arbeit zu Strukturen und Akteuren der Altenpflege in Deutschland. Ihre derzeitigen Lehr- und Forschungstätigkeiten konzentrieren sich auf den demographischen Wandel und seine Auswirkungen auf Wirtschaft und sozialen Dienstleistungssektor, aktuelle Entwicklungstrends in der Sozialwirtschaft, insbesondere Social Entrepreneurship, sowie Wohlfahrtsverbände sowie lokale Sozialpolitik.

www.hs-koblenz.de/schneiders

\author{
Der seit einiger Zeit in der sozialwirtschaftlichen \\ Diskussion verwendete Bezeichnung des "Social \\ Entrepreneurships" bezieht sich vor allem auf \\ angelsächsische und ökonomisch geprägte Definitionen. \\ Ein neue Veröffentlichung sucht nach einer \\ möglichen ethischen Fundierung des Phänomens.
}

Das in der Monographie, die Überarbeitung einer an der Hochschule Koblenz eingereichten Master-Thesis, von Sina Slottke thematisierte Phänomen des Social Entrepreneurship erfreut sich seit mittlerweile einigen Jahren massiver Aufmerksamkeit in Politik und Öffentlichkeit.

Die wissenschaftliche Diskussion um die gesellschaftliche Relevanz des "Social Entrepreneurships" hat sich in Deutschland im internationalen Vergleich jedoch erst relativ spät entwickelt (vgl. als einen ersten umfassenden Überblick die Beiträge in Hackenberg/ Empter 2011 und Jähnke/Christmann/ Balgar 2012 sowie die kursorische Analyse von Rummel 2011). Während die dort versammelten Beiträge aus wissenschaftlicher und eher anwendungsbezogener Perspektive u. a. auf gesellschaftliche Rahmenbedingungen, Finanzierungsbedingungen und Praxisbeispiele eingehen, konzentriert sich Sina Slottke mit der Frage nach der Wertorientierung von Social Entrepreneurships auf eine bis dato noch nicht systematisch bearbeitete Fragestellung.

Die Frage nach der ethischen Orientierung von Sozialunternehmen, also der Frage nach dem "Sozialen « ist von dauerhafter Relevanz - insbesondere in Zeiten fortschreitender Ökonomisierung des Sektors - und markiert insofern wie von Slottke zutreffend diagnostiziert eine "Lücke im Theoriegebäude « ( S. 14) des Phänomens des Social Entrepreneurships.
Insofern ist das Anliegen der Arbeit erfreulich, einen Bezug zur »Sozialen Arbeit herzustellen « (S. 13) oder wie später deutlich werden wird, den Wertekanon der Sozialen Arbeit als Legitimationsbasis zu nutzen. Angesichts der Neuartigkeit und Hybridität des Social Entrepreneurships ist die vorgelegte ausführliche Darstellung und Diskussion möglicher historischer Bezüge für einen Einsteiger in die Thematik hilfreich.

Da Slottke ausgehend von dem englischen Begriff hier vor allem auf Literatur angelsächsischer Provenienz Bezug nimmt, bleiben spezifisch deutsche Entwicklungen wie die der deutschen Wohlfahrtsverbände (als vielleicht erste »Sozialunternehmer«) unberücksichtigt.

Im Anschluss an den kursorischen Überblick über mögliche historische Bezüge, widmet sich die Monographie dem Begriff des »Social Entrepreneurships « und gibt einen Überblick über vorhandene Definitionen und Definitionsversuche. Auch hier liegt der Fokus auf der Auswertung angelsächsischer Literatur, was angesichts der noch spärlichen deutschsprachiger und auf Deutschland fokussierender Literatur nachvollziehbar ist.

Der von Slottke aus der internationalen Debatte destillierte präsentierte Definitionsversuch (S. 47) bleibt ähnlich wie andere bislang vorliegende deutsche Definitionen leider unvollständig, da die als zentrales Element 
von Social Entrepreneurship genannte "wirtschaftliche Komponente " nicht näher erläutert wird.

In Bezug auf die hier im Mittelpunkt stehende Fragestellung nach der ethischen Fundierung werden zwei Varianten von Social Entrepreneurship theoretisch hergeleitet und unterschieden: ei-
Human Rights Profession skizziert Slottke schließlich einen möglichen ethischen Bezugsrahmen für Social Entrepreneurs.

Die Schwierigkeiten der Fassung des »multidimensionalen Phänomens" resultieren vor allem aus der nicht konsequent durchgehaltenen analytischen

\section{"Manche sSocial Entrepreneuersı distanzieren sich dezidiert von der Sozialen Arbeit»}

ner auf »Gemeinschaft ausgerichtete[n] Variante " und einer "persönlichkeitszentrierten Variante« (62). Es wird zu Recht darauf hingewiesen, dass es sich bei diesen Varianten um Extrembeispiele handelt, die in der Praxis von sehr vielen Zwischenformen ergänzt werden - eine empirische Fundierung dieser These bleibt künftigen Untersuchungen vorbehalten.

Aus Sicht der Autorin »erscheinen die Menschenrechte als ethischer Bezugsrahmen für Social Entrepreneurship " als "alternativlos « - eine Aussage, der angesichts ihrer Allgemeingültigkeit sicher von jedem Akteur zugestimmt werden kann. Aus der Perspektive Sozialer Arbeit als

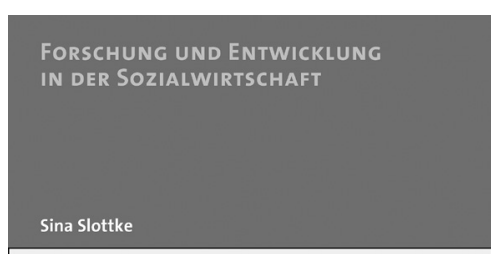

Social Entrepreneurship als multidimensionales Phänomen

Ansatzpunkte zur Theorieentwicklung aus der Perspektive Sozialer Arbe

Nomos

Sina Slottke: Social Entrepreneurship als multidimensionales Phänomen. Ansatzpunkte zur Theorieentwicklung aus der Perspektive der Sozialen Arbeit. Nomos, Baden-Baden 2013. 104 Seiten. 19,- Euro. ISBN 978-3-8487-0069-1.
Trennung von (Organisations-) Struktur und Handlungsstil der Akteure. Hier stößt Slottke an ähnliche Grenzen wie andere Autoren, da eine systematische Trennung von Organisations- und Rechtsform sowie Handlungsstil empirische Daten erfordert, die bislang nur vereinzelt erhoben wurden und von der Autorin nicht berücksichtigt werden konnten (Grohs/Schneiders/ Heinze 2013). Eine Übertragung der internationalen Definitionsversuche muss darüber hinaus an der fehlenden Berücksichtigung der institutionellen Rahmung scheitern, also an der Frage, inwiefern ein in liberalen und defizitären Wohlfahrtsstaaten (USA, Entwicklungsländer) entwickeltes Konzept auf einen etablierten Wohlfahrtsstaat wie Deutschland übertragen werden kann.

Es ist ein besonderes Verdienst des vorliegenden Bandes, das Phänomen des Social Entrepreneurship aus der Perspektive der Sozialen Arbeit zu diskutieren. Vor allem, da die sich selbst als "Social Entrepreneurs" bezeichnenden Akteure oftmals keinen direkten Bezug zur Sozialen Arbeit aufweisen oder sich von diesem teilweise sogar dezidiert abgrenzen. Auch die wissenschaftliche Debatte wird bislang von Wirtschaftsund Politikwissenschaftlern sowie Soziologen dominiert.

Offen bleibt, inwiefern die theoretisch-konzeptionellen Ausführungen empirisch fundiert werden können und ob die Social Entrepreneurs (wie von einigen so postuliert) einen ganz neuen Typ sozialer Dienstleistungsunternehmen darstellen und gegebenenfalls die überwiegend in traditionellen Strukturen der Freien Wohlfahrtspflege agierende Soziale Arbeit ihrerseits von Impulsen profitieren könnte.

\section{Literatur}

Grohs, Stephan/Schneiders, Katrin/Heinze, Rolf G. (i.V.): Mission Wohlfahrtsmarkt. Institutionelle Rahmenbedingungen, Strukturen und Verbreitung von Social Entrepeneurship in Deutschland, Baden-Baden: Nomos.

Hackenberg, Helga/Empter, Stefan (Hg.) (2011): Social Entrepreneurship - Social Business:

Für die Gesellschaft unternehmen, Wiesbaden: VS Verlag.

Jähnke, Petra/Christmann, Gabriela B./Balgar, Carsten (Hg.) (2012): Social Entrepreneurship. Perspektiven für die Raumentwicklung,

Wiesbaden: VS.

Rummel, Miriam (2011): Wer sind Social Entrepreneurs in Deutschland? Versuch einer Profilschärfung, Wiesbaden: VS.

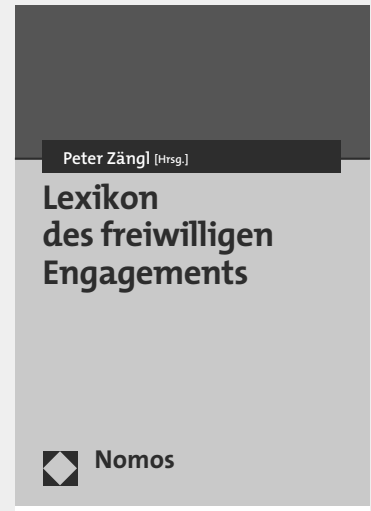

\section{Lexikon des freiwilligen} Engagements

Herausgegeben von

Peter Zängl

2014, ca. 500 S., brosch., ca. 49,-€ ISBN 978-3-8329-7248-6

Erscheint ca. März 2014

Dieses Lexikon systematisiert und definiert rund 300 Schlüsselbegriffe, erläutert diese anhand von praktischen Beispielen und ist Wegweiser für weitere Recherchen.

www.nomos-shop.de/14401

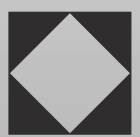

Nomos 